\title{
La primera conformación de una audiencia infantil y juvenil para la televisión en España (1958-1968)
}

\author{
María Antonia Paz Rebollo \\ Universidad Complutense de Madrid \\ mapazreb@ucm.es \\ Lizette MARTíNEZ VALERIO \\ Universidad Complutense de Madrid \\ lmvalerio@pdi.ucm.es
}

Recibido: 04/02/2014

Aceptado: 10/03/2014

\section{Resumen}

Se analiza cómo se creó la primera audiencia infantil y juvenil en Televisión Española en los primeros años de emisión. Para ello se tienen en cuenta todos los programas de producción propia dirigidos a los más jóvenes, así como su distribución por franjas horarias y días de la semana. También se estudian los contenidos de estos programas, que se han visionado, para valorar su temática, mensajes emitidos y objetivos de los mismos. Se concluye que las necesidades de una televisión pública con monopolio condujeron a dar prioridad a programas dirigidos a toda la familia y que los espacios infantiles se supeditaron a esta exigencia. Se buscó sobre todo entretener a los más jóvenes, aunque no se desaprovechó la oportunidad de educar y adoctrinar en valores religiosos y patrióticos.

Palabras clave: Audiencia infantil, audiencia juvenil, televisión española, programación, franquismo.

\section{Shaping the children's and youth audience for television in Spain (1958-1968)}

\begin{abstract}
The present work discusses how it was created the first children's and youth audience in Televisión Española during its first years of broadcasting. In order to do this, the investigation studies all own production programs addressed to the younger audience, as well as their distribution by time slots and days of the week. It also studies the contents of these programs, which were viewed in order to assess its subject, message and objectives. It is concluded that the needs of a public television monopoly led to prioritize programs for the whole family, and children's spaces were subordinated to this requirement. The goal was to entertain, especially the younger ones, without missing the opportunity to educate and indoctrinate religious and patriotic values.
\end{abstract}

Keywords: children's audience, youth audience, Spanish televisión, programming, francoism.

Referencia normalizada: PAZ REBOLLO, María Antonia y MARTÍNEZ VALERIO, Lizette (2014): "La primera conformación de una audiencia infantil y juvenil para la televisión en España (1958-1968)". Estudios sobre el Mensaje Periodístico, Vol. 20, Núm. especial, pp. 43-58. Madrid, Servicio de Publicaciones de la Universidad Complutense.

Sumario: 1. Objetivos y descripción de la metodología. 2. Claves de la programación. 3. Los primeros tanteos (1958-1963). 4. Nuevas propuestas (1964-1968); 4.1. Los jóvenes entran en escena; 4.2. Modelos a seguir. 5. Conclusiones. 6. Referencias. 7. Archivos. 8. Anexo: Programas infantiles y juveniles de TVE (1958-1968). 


\section{Objetivos y descripción de la metodología ${ }^{1}$}

El objetivo de este artículo es analizar cómo se formó la primera audiencia infantil y juvenil en Televisión Española a lo largo de los primeros años de emisión. Para ello se examinan qué contenidos de producción propia se ofertaron entre 1958 y 1968, cuál fue su evolución y su presencia en los diferentes días de la semana y, en la medida que de lo posible, cómo fueron recibidos por los espectadores más jóvenes. El tema reviste una gran importancia porque permite valorar el concepto que de los niños se tenía en esos años y también el papel de la televisión en sus necesidades educativas y de entretenimiento. Se demostrará que estos programas pretendían sobre todo entretener, pero al mismo tiempo se otorgó a la televisión la misión de inculcar a los niños las normas de los adultos.

La bibliografía no ha prestado apenas atención a la programación ni al contenido de los programas de la televisión española de entonces: la programación infantil no es una excepción. Salvo Manuel Palacio (Palacio, 2005) y alguna otra investigación (Baget, 1993 y 1999), la mayor parte se apoya en la memoria de los responsables de televisión, en la prensa de la época y en algunas secuencias aisladas que se localizan en internet. Lorenzo Díaz (Díaz, 1994) hizo un estudio desde la intrahistoria y Jesús García analizó la política cultural en RTVE durante el franquismo (García, 1980), mientras que Sira Hernández se ha centrado en los documentales de divulgación histórica (Hernández, 2008). El catálogo de programas infantiles elaborado por Manuel María González (González, 2011) contiene errores, tanto en las fechas de emisión y finalización de programas, como en la clasificación de los mismos. Más fiable es la base de datos elaborada por Tamara Antona para la realización de su tesis doctoral, pero en la misma no consta el contenido de los programas.

Ha sido por tanto necesario localizar todos los programas infantiles y juveniles de producción propia emitidos entre 1958 y 1968 a través de la información televisiva publicada por $A B C$ y La Vanguardia, en su hemeroteca online, y por la revista Tele Radio. Además se han realizado consultas (los títulos de todos los programas) en la base de datos de TVE -ARCA- Este material se ha completado con los documentos que se conservan en el Archivo General de la Administración sobre las emisiones diarias de TVE referidas a este periodo.

El estudio se inicia el 1 de enero de 1958 porque fue entonces cuando comenzó a publicarse en prensa general la programación de TVE y se cierra el 31 de diciembre de 1968 porque se produjo un cambio importante en la configuración de los programas infantiles: disminuyeron los estrenos, pero los programas se prolongaron durante más tiempo y cambiaron los contenidos. Coincide con la última etapa del mandato de Jesús Aparicio-Bernal Sánchez, que fue el director general de Radiotelevisión que más años estuvo en el cargo durante el franquismo (1964-1969).

\footnotetext{
Esta investigación se ha realizado dentro de las actividades del proyecto de investigación "Televisión y Cultura popular durante el franquismo: Programación, programas y consumo televisivo (1956-1975)", ref. HAR2011-27937; y dentro del Grupo Complutense de Investigación consolidado Historia y Estructura de la Comunicación y el Entretenimiento $\left(\mathrm{n}^{\mathrm{o}}\right.$ 940439), de la Universidad Complutense de Madrid (España).
} 
Sólo se han incluido los programas cuya temática estaba dirigida a este público específico y que se emitieron desde las 16,30/ 17,00 (hora de salida de los colegios) hasta la emisión, a las 20'55 horas aproximadamente, del programa Vamos a la cama, en el que unos dibujos animados recomendaban a los espectadores más jóvenes acostarse. También se ha tenido en cuenta la programación infantil del fin de semana, tanto matutina como vespertina.

No se analizan ni dibujos animados ni telefilms extranjeros, porque se atiende sólo a la producción propia, como se ha señalado. Tampoco los programas con un carácter eminentemente educativo (enseñar un idioma, reforzar un ciclo de enseñanza) puesto que exigen, por su amplitud, otra investigación. Sí se tienen en cuenta los espacios destinados a los diferentes grupos de edad: desde Jardilín a Cesta y puntos.

Con este material se ha elaborado una base de datos compuesta por 77 registros en la que se han incluido el nombre del programa, tipo de contenido ${ }^{2}$ (deportivo, educativo, variedades, circo, concurso, divulgativo, ficción, juegos, informativo, humor, musical, contenedor, religioso), la fecha de inicio y finalización de la emisión, hora y día de emisión, periodicidad, nombre del director, presentador, guionista, realizador, palabras clave con el contenido y observaciones. Estos datos han servido para contabilizar el número de estrenos por años, la duración media de los programas -con el fin de valorar su continuidad en la parrilla- y su presencia en los diferentes días de la semana, distinguiendo especialmente entre días laborables y fines de semana. También se ha tenido en cuenta qué tipo de contenidos han sido predominantes en la programación infantil y cuáles han ocupado un lugar minoritario.

Finalmente, se han visionado 18 programas infantiles y juveniles que de esta época se conservan en el archivo de TVE con el fin de analizar los contenidos, los personajes que aparecen, la función de los presentadores, la actividad de los niños dentro del programa o desde sus casas y los enseñanzas que se difunden. A través de la prensa general ( $A B C$ y La Vanguardia) y la especializada (Tele Radio) se ha recabado información sobre el contenido de los programas y sobre la opinión que de estos espacios y, en general de la programación infantil y juvenil, se publicó entonces. En este sentido, las cartas al director han proporcionado pistas interesantes sobre la recepción de estos espacios.

Las conclusiones ponen de manifiesto que en estos años se creó una programación infantil, pero no fue ni amplia ni exclusiva puesto que los contenidos de muchos de los programas iban dirigidos a toda la familia. Se observa un tratamiento del niño como un adulto pequeño al que la televisión trata de entretener. A partir de 1964, los contenidos intentaron adaptarse algo más a los gustos y a las diferentes edades de los niños. No obstante, el espíritu patriótico y la formación doctrinal cristiana estuvieron presentes en la oferta televisiva a lo largo de todos estos años.

2 En esta clasificación se ha tenido en cuenta la denominación que el departamento de producción de entonces otorgaba a cada programa y, en caso de ausencia, la que aplicaba la prensa de la época. 


\section{Claves de la programación}

Pueden distinguirse dos periodos en estos años. El primero corresponde al arranque (1958-1963). Los problemas técnicos y de producción acaparaban gran parte de la atención de los profesionales del medio, puesto que se emitía en directo. Las emisiones comenzaban a las nueve y media de la noche y duraban tres horas (Bethancourt, 1996: 61). Los programas infantiles consistían entonces en actuaciones circenses en el estudio, teatro de marionetas (cámara fija delante del escenario) y dibujos animados norteamericanos -también series como Rin Tin Tin- ya enlatados. En 1958, comenzaron los concursos de producción propia (Primer aplauso) y los programas religiosos para los más pequeños (Lecciones de catecismo). TVE imitó la tradición radiofónica, sobre todo la de Radio Nacional de España que se preocupó especialmente por este sector de la población (Revista de la Opinión Pública, $\mathrm{n}^{\circ} 1,1965: 293$ ).

En 1964 se inició una nueva fase con Jesús Aparicio Bernal como director general de RTVE. Fueron los años en los que se inauguraron los estudios de Prado del Rey y la televisión expandió su presencia social. También se llevaron a cabo cambios importantes en los contenidos de los programas para darles "un matiz más acorde con la mentalidad de los niños" (La Vanguardia, 25 de noviembre de 1965) y se organizaron congresos sobre la Televisión infantil y juvenil que, aunque sólo fueron dos (en 1964 y 1965), dan idea de la preocupación por el tema.

No obstante, la presencia de programas infantiles y juveniles no fue destacada en este periodo. De las nueve horas de emisión, por término medio que tenía TVE en estos años, se dedicaron, a los más jóvenes, 45 minutos, más o menos, entre 1958 y 1963; aunque esta media diaria se incrementó a una hora y 10 minutos, entre 1964 y 1968. Hay que tener en cuenta que los niños eran los miembros de la familia que pasaban más tiempo ante el televisor (Revista Española de la Opinión Pública, $\mathrm{n}^{\circ}$ 2, 1965: 215).

En once años se estrenaron 77 programas: 34 en el primer periodo y 43 en la segundo. Estos programas permanecieron en pantalla una media de un año, aunque hubo excepciones: Chavales duró 3 años; Recreo, 4 y Cesta y puntos, 6. Uno de los programas más longevo fue Misión Rescate.

Temáticamente, predominaban los programas dedicados a la ficción, es decir, al entretenimiento, aunque, como se explicará, muchas historias iban cargadas de adoctrinamiento. El segundo lugar lo ocupaban los divulgativos (ver Tabla 1) lo que da una idea del afán de TVE por compaginar, en la programación destinada a los más jóvenes, entretenimiento y formación. Los concursos y variedades se situaban en cuarto y quinto lugar y los contenedores comenzaron a despuntar, aunque no alcanzaron protagonismo hasta el periodo posterior (1969-1975). El resto tuvieron poca relevancia numérica, pero con matices. Por ejemplo, los religiosos no fueron abundantes, pero se mantuvieron a lo largo de todos estos años, así como el circo que alcanzó gran éxito en los últimos años del franquismo con las actuaciones de los llamados "payasos de la tele" (Gaby, Fofó y Miliki). No cuajaron los informativos ni los programas de humor, éstos últimos porque en realidad eran programas destinados para los mayores, en un supuesto horario infantil (Mundo ligero, El barquito de papel). Los espacios deportivos curiosamente también fueron escasos. 
Tabla. 1. Clasificación por contenidos de los programas infantiles de TVE (1958-1968)

\begin{tabular}{|c|c|}
\hline Contenido del programa & $\mathbf{N}^{\mathbf{0}}$ de programas emitidos \\
\hline Ficción & 17 \\
\hline Divulgación & 13 \\
\hline Concursos & 9 \\
\hline Variedades & 8 \\
\hline Educativos & 6 \\
\hline Contenedor & 5 \\
\hline Juegos & 5 \\
\hline Música & 4 \\
\hline Religiosos & 3 \\
\hline Deportivos & 3 \\
\hline Humor & 2 \\
\hline Circo & 1 \\
\hline Informativos & 1 \\
\hline
\end{tabular}

Fuente: Elaboración propia

En el primer periodo, había programas infantiles los fines de semana y los jueves por la tarde, porque en algunos centros escolares no había actividad docente, con programas como A la rueda, rueda, Recreo y Chavales. A partir de 1964, se extendieron a todos los días de la semana, aunque desaparecieron prácticamente de los domingos por la tarde como consecuencia de las retransmisiones de fútbol (Bonaud, 2008). En la mañana de los domingos tampoco se programaban espacios infantiles, porque se entendía que se llevaba a cabo la práctica religiosa. La mayoría de los programas eran semanales. Sólo Cuento infantil y Jardilín aparecían tres días a la semana.

A pesar de que la revista Tele Radio presentaba la programación fragmentada en tres franjas ("Sobremesa", "Infantil" y "Noche"), lo cierto es que los programas no se ajustaban a esa realidad: a veces los dibujos animados se colocaban en el llamado horario de noche; mientras que programas para mayores aparecían en horario infantil.

\section{Los primeros tanteos (1958-1963)}

En la inauguración de un Curso sobre Radio, Televisión y Teatro infantil, celebrado en mayo de 1959, el Secretario General de TVE, Antonio Farré de Calzadilla, explicó que, en los programas infantiles, debía haber fantasía, para desarrollar la imaginación de los niños, pero también temas costumbristas y populares, para que los niños se acercasen a la realidad circundante; y temas heroicos, legendarios y hagiografías "que han de contribuir a completar su formación, inculcándoles sentimientos patrióticos y religiosos de incuestionable transcendencia" (Tele Radio, 4 de mayo de 1959). Esta fue la línea de actuación en los contenidos televisivos infantiles.

Las cartas que los lectores enviaban a la revista Tele Radio hacen pensar que a los niños nos le gustaban estos programas (Tele Radio, 4 de mayo de 1959), que eran rutinarios (Tele Radio, 6 de junio de 1960), con gazapos (Tele Radio, 10 de agosto de 1964) y no se prestaba atención a los jóvenes (Tele Radio, 27 de junio de 1960). 
En estos años los programas destinados al entretenimiento fueron los más numerosos: variedades y ficción, especialmente las primeras que consistían en actuaciones de payasos y otros números circenses, grupos musicales, marionetas, magia, cuentos, aventuras, imitaciones, mimos y concursos. Se iniciaron en 1959 con el programa No apto para mayores que se emitía los sábados. Gracias a su buena acogida inicial, la oferta se incrementó, tras su finalización en septiembre de 1959, a los jueves (A la rueda, rueda) y a los domingos (primero con Gente menuda y después con Tíovivo).

La renovación de este tipo de espacios llegó los jueves con Recreo y con Chavales (un año después), cuyo guión y dirección corrió a cargo de Juan Francisco Puch. Estaban orientados a una audiencia familiar. Sus presentadores, Boliche y Chapinete, se hicieron muy famosos, junto a Cheles, Polilla y el Tío Chus que, en Chavales, amenizaban las actuaciones y las diversas actividades propuestas, como, por ejemplo, confeccionar caretas o dar lecciones de gestos o actitudes, teatro de marionetas, una orquesta infantil, lección de gimnasia a cargo de escolares madrileños y las aventuras de la burrita Currita. Incorporaron una orquesta propia, la de Manolo Gracia, y contaban con la presencia de niños (también colegios) en el estudio. Chavales pasó a denominarse Antena infantil en noviembre de 1965.

Aunque los números de circo eran habituales en estos programas, se ensayó la fórmula de centrar todo un espacio en este espectáculo. El resultado fue Silla de pista (1962-1963), una oferta dominical que daba continuidad a las primeras emisiones televisivas circenses, puesto que el circo era considerado el programa infantil por excelencia.

El primer contenedor se llamó Fiesta con nosotros y se emitió los domingos, entre 1962 y 1965. Herta Frankel y sus marionetas constituían el eje central de la emisión compuesta de cuentos, concursos, juegos, actuaciones y las aventuras de Tintín y los Picapiedras. Su objetivo también era hacer pasar a los niños una tarde divertida

La ficción para niños en este primer periodo tuvo como protagonistas a las marionetas y el referente más conocido fue la ya mencionada Herta Frankel. Representaba el papel de madre que dialogaba, aconsejaba, incluso regañaba a sus hijos (marionetas). Estas representaban aventuras e historias sencillas. Herta preparaba sus actuaciones para llegar a los niños: "leo todos los cuentos infantiles que se publican, y hablo continuamente con los niños, les pregunto sus cosas, quiero saber sus gustos, estudio sus reacciones..." (Tele Radio, 27 de enero de 1964). El resultado eran unos personajes que exploraban el mundo infantil, aunque algunas veces hacían pensar a los mayores.

De estructura muy similar (música e historias con marionetas para los pequeños) se ofreció Walter y la familia corchea (1960), donde Pedrito y todos sus parientes con el hada Alicia y los muchachos músicos vivían emocionantes aventuras.

La escenificación y narración de historias también constituyeron un elemento fundamental de la programación infantil a lo largo de estos años: la fórmula se tomó de la radio. En Un mundo para ellos (1963), escrito, dirigido y presentado por Salvador Salazar, el Tío Salva, apodado el amigo de los niños, contaba una historia siempre interesante e instructiva. En diez minutos se narraba un cuento clásico en Cuento infantil: Alicia en el país de las maravillas, La ratita presumida, Simbad el marino, entre otros muchos. 
En Teatro infantil, titulado a veces como juvenil e infantil, se dieron a conocer obras clásicas como El principito, El lazarillo de Tormes, El soldado fanfarrón, basada en una obra de Plauto, o La Tina de la colada, obra francesa del siglo XV, entre otras. También se representaron obras de autores españoles en las que los protagonistas eran niños, como El hombre de las cien manos (historia de un niño sordomudo de ocho años maltratado por los habitantes de su pueblo), El cartero del rey (un niño enfermo que sueña con ser cartero), Zuecos y Naranjas (la amistad de un niño danés y uno español), El chico (el ascenso por méritos propios de un chico de mozo a encargado de una ferretería). Dentro de la ficción, resultó novedoso Rumbo sur (1963) porque escenificaba aventuras reales, por ejemplo, la conquista del Everest.

No faltó adoctrinamiento religioso y moral en estos programas. Por ejemplo, en historias que narraban la conversión al catolicismo (Recreo) o de milagros divinos ( $\mathrm{Te}$ atro infantil). En otros (Silla de pista) se incluyeron concursos como el de "los niños y niñas gordos" que mostraban a todos los espectadores el inicio de la bonanza económica en el país.

Los concursos, presentes de forma destacada en la programación destinada a adultos, fueron bien recibidos por los espectadores más jóvenes. Se estrenaron dos dedicados al mundo de la música: Primer aplauso y Concertino. El primero, dirigido en realidad a todos los públicos, trataba de promover a artistas noveles (reaparecerá en 1966 con el mismo título). La edad mínima para participar era de 15 años. El ganador se incorporaba al elenco de artistas profesionales que cubrían la programación musical de TVE. Concertino (1963-1964) era en realidad un espacio de variedades, cuyo formato se compró a la RAI (Carreras, 2011), que buscaba la participación de los espectadores con un concurso de canciones infantiles famosas. En él Torrebruno inició su carrera televisiva sin mucho éxito (Baget, 1993: 134).

Premio al más listo tenía un contenido cultural, igual que Sobresaliente cuyo eslogan era "el concurso para los sabios de cada colegio", mientras que La vida y el juego, consistía en una rueda de prensa en la que seis niños y niñas hacían preguntas a un niño famoso y recibían el carné de "Mini periodistas de honor". Después se ofrecía un concurso filmado en el que los espectadores veían ocho objetos en cincuenta y ocho segundos y dos fotografías de famosos, convenientemente embozados. Mandaban una carta con la solución y recibían el juguete que pidiesen. El objetivo no era sólo jugar a ser mayores (periodistas), sino interesar a los niños por algo y alguien y, a través del juego, enseñarles las extraordinarias posibilidades del diálogo. Duró sólo un año.

En este periodo se pusieron en marcha iniciativas interesantes, aunque algunas no tuvieron continuidad. Por ejemplo, el Telepequediario, que durante un año ofreció noticias a los pequeños todos los sábados. Durante el verano de 1961, se emitió Torneo (el título se retoma en 1975), dedicado a la competición deportiva infantil y juvenil, que entonces no cosechó ningún éxito, igual que los programas de humor El barquito de papel y Mundo ligero (al menos entre los más pequeños). Ambos fueron escritos por Vadorrey y Chumy Chumez con el humor sarcástico que les caracterizaba. En realidad, estaban destinado a los adultos, pero emitidos en horario infantil.

Los programas divulgativos, religiosos y los dedicados a juegos sí encontraron un hueco en la programación infantil posterior. Divulgativos fueron Lecciones de cosas 
y Visado para el futuro. El objetivo de este último era dar a conocer "las nuevas ideas, los últimos descubrimientos científicos", pero no descuidó el orden espiritual al hablar del futuro, de hecho, el padre Urteaga intervino en más de una ocasión. Se trataba de poner al alcance del espectador medio, "cuando un hombre de hoy medianamente culto, necesita saber". También de despertar en los jóvenes vocaciones científicas, haciendo hincapié en los aspectos más atractivos de la ciencia. Se recurría para ello a la ficción, la dramatización y la espectacularidad (Ortega y Albertos 1998:69). Se incluye en el programa infantil, pero en ocasiones aparece en la programación de noche. Se realizaba en Barcelona y estaba presentado Luis Miravitlles.

Además de Lecciones del catecismo (1959), espacio en el que el padre Vázquez aleccionaba a los niños los jueves sobre religión, se realizó otro programa también con contenido religioso/moral titulado Sólo para menores (o Sólo para menores de 16 años) que estuvo en antena entre 1962 y 1966. Unos jóvenes interpretaban una pequeña historia (no siempre la estructura del programa era así) que servía para que el padre Jesús Urteaga comentase un tema, como el miedo, la tenacidad, policías y ladrones, la familia, las vacaciones, entre otros. Su lema era "Siempre alegres para hacer felices a los demás". Sólo hubo un espacio dedicado a enseñar a jugar. Se llamó $M u$ chachos al aire libre, y se emitió los domingos, a las 18,00, durante el mes de vacaciones.

\section{Nuevas propuestas (1964-1968)}

En este periodo se estrenó el spot publicitario Vamos a la cama, de 55 segundos de duración, protagonizado por la familia Telerín que alcanzó un éxito sin precedentes. La canción fue compuesta por Antonio Arteta y los protagonistas fueron creados por José Luis Moro, propietario, junto con su hermano Santiago, de la agencia de publicidad de su nombre (Fernández, 2007: 103). Se trataba de una separata, es decir, servía para distinguir una programación de otra: la que podían ver los niños y la que no, lo que da una idea de que la programación infantil comenzaba a adquirir una cierta diferenciación.

Se organizó igualmente el Club infantil de TVE para contar con los niños, como socios, en diferentes actividades, concursos, actuaciones, etc. Era evidente la intención de atraerse y fidelizar con esta iniciativa a la audiencia infantil y juvenil. Los resultados fueron positivos: en 1967, por ejemplo, contaba con más de 100.000 suscripciones (Granados, 1966).

Los programas divulgativos y educativos experimentaron un fuerte impulso, aunque los padres consideraban que la televisión era indiferente para los estudios de sus hijos y los niños entendían que la televisión era un instrumento de evasión (Revista Española de la Opinión Pública, nº 1,1965: 295).

La radio dejó de ser el único modelo a seguir y se buscó inspiración en televisiones extranjeras. Pepe Palau, por ejemplo, consiguió los derechos de explotación de algunos programas de la televisión americana. Uno de ellos fue Clan familiar, un concurso emitido en la tarde los sábados en el que sólo participaban familias numerosas. Se proyectaba un film corto de cine mudo o un informativo de TVE y se hacían cinco preguntas para medir su capacidad de atención y memoria. Tuvo una buena acogida. 
También Jardilín se inspiró en la serie de televisión norteamericana Romper Room, destinada a los más pequeños. La acción se desarrollaba en un aula de guardería. La presentadora -Charo Tijero e Isabel Osorio sucesivamente- hacía las veces de maestra de seis niños (3 niñas y 3 niños), de 4 a 6 años. Durante media hora, los niños pintaban, aprendían a hacer manualidades, ejercicios físicos y cantaban. Se invitaba a los niños que estaban en su casa a participar. El objetivo era que aprendiesen a "ser buenos" y para ello se utilizaba al Señor Si, que era un abejorro sonriente y el Señor No era un abejorro enfadado que era mostrado por la maestra cuando los niños no hacían algo bien. En la última parte del programa la maestra contaba un cuento con moralina (muchos eran parábolas religiosas) que los niños no siempre entendían. Se despedían colocando un espejo "mágico" delante de su cara con el que supuestamente veían a los espectadores. El programa tuvo muy buenas críticas. En 1968 fue sustituido por Clarín, con Charo Tijero también como presentadora. Este nuevo programa enseñaba a los niños juegos para realizar en casa y al aire libre, acompañados de una canción original. Los pequeños también participaban en su casa enviando cartas a TVE votando su juego favorito.

\subsection{Los jóvenes entran en escena}

Se iniciaron programas destinados a los jóvenes, entre 15 y 18 años. Habla contigo era un espacio breve en el que el padre Urteaga mantenía un breve coloquio con los espectadores juveniles sobre temas teológicos y morales. Se trataba de formar la conciencia juvenil en torno a problemas religiosos tanto en su vertiente personal como social. Tenemos la palabra también se ocupaba de las preocupaciones de la juventud que vivía en el campo: Por ejemplo, Nosotros no nos aburrimos, Tenemos una canción, ¿Qué hacemos con los viejos?, El Hambre, Madre y amiga, El baile, Los vicios, Chicos y chicas, La justicia, etc. (AGA, Caja 35692). En un coloquio en el estudio se confrontaba el punto de vista de los jóvenes y de los adultos.

Muy similar fue Objetivo: una inquietud, también escrito por Juan Mayor de la Torre, o ¿Cuál es tu final? presentado por el actor Jaime Blanch y Gloria Roig. Estas producciones televisivas no terminaron de cuajar, porque "aburrían", según algunas cartas publicadas en la revista Tele Radio.

La segunda propuesta para la gente joven se centró en la música moderna, del tipo Todos somos jóvenes. Romano Villalba fue el guionista y coordinador del espacio. Estaba dirigido a chicos y chicas entre 13 y 18 años, aunque en realidad era para todos los públicos (Tele Radio, 15 enero de 1968). En él actuaban conjuntos como Los Relámpagos, Los Vampiros o Los Canarios, entre otros. Participaban también los espectadores solicitando, a través del correo, la aparición de un conjunto o respondiendo a las preguntas que sobre pintura y música se planteaban. Se comentaban noticias, que se ilustraban con fotos, de las más importantes aparecidas en la prensa, tanto deportivas, como culturales o políticas.

En este periodo TVE inició la realización de aventuras seriadas para los jóvenes. En Santi, botones de hotel, el protagonista era un joven dispuesto a meterse en todo tipo de líos. Protagonizaba historias de espías, complots, marcianos, incluso sabotaje en un encuentro de fútbol entre el Barcelona y el Real Madrid. Antoñita la fantástica se dirigía a niñas de entre doce y quince años. Era el personaje principal de una co- 
lección literaria escrita por Borita Casas, que también escribió el guión de la serie televisiva. Se emitía la tarde de los domingos y la realización corrió a cargo de Pilar Miró. En esta versión para TVE, Antoñita era una joven ye-ye, minifaldera, que no quería engordar, pero que seguía siendo una chica inocente y tan fantástica como cuando era niña.

Patio de corredor fue también una serie de aventuras escrita por Montserrat del Amo, basada en una novela que, con el mismo título, figuró en la lista de honor del premio internacional Andersen. Estaba ambientada en un patio donde vivía Maruja, una chica inteligente, estudiosa y con afán de superación que deseaba un porvenir mejor, un trabajo más interesante, una vida más elevada, pero no contaba con medios para conseguirlo. A través de los diferentes episodios comprendió que el valor de un ser humano está más en lo que hay dentro que en la posición social que ocupa.

También en este periodo, y como novedad, se crearon espacios para promover competiciones colegiales. En este sentido, el programa con más éxito fue el concurso Cesta y puntos, versión televisiva de un espacio radiofónico ideado y presentado por Daniel Vindel. La dinámica del programa era similar a la de un partido de baloncesto. El público (familiares y profesores) en el estudio aplaudía y cantaba canciones típicas de su localidad cuando su equipo acertaba la respuesta. Los chicos se sentaban en pupitres: dos eran los delanteros, dos los defensas, un pivot y tres suplentes. Las preguntas se alternaban y si un equipo fallaba, rebotaba al pivot contrario. Los concursantes podían hacer falta y personal. La temática de las cuestiones era muy variada: desde latín, historia, matemáticas, física, biología, incluso religión, todo ello a nivel de bachillerato. Se combinaba entretenimiento y divulgación cultural y era visto por todo tipo de público.

El éxito de Cesta y puntos influyó en otros concursos que se especializaron en ambientes concretos, por ejemplo, La mar, ese mundo maravilloso, aunque tuvo más repercusión Misión rescate, un concurso escolar que se inició en RNE, en el que un grupo de niños, dirigido por un profesor o un párroco, descubrían, localizaban y valoraban obras de arte o restos arqueológicos. Su labor permitió catalogar una parte importante del patrimonio de cada región.

\subsection{Modelos a seguir}

La educación de los niños y los jóvenes siguió presente en la programación. En este segundo periodo, los objetivos fueron tres: uno, formar a los niños en valores religiosos, morales, sociales, políticos concretos utilizando modelos de comportamiento; otro, orientar a los estudiantes hacia la formación profesional; y finalmente, enseñarles a ocupar su tiempo libre de forma útil, a tener un hobby.

Respecto a los primeros, destacó Escuela de campeones. Tenía un carácter didáctico: enseñaba a los niños y a los jóvenes lo que significaba el esfuerzo, el sacrificio y la renuncia. Consistía en que una figura destacada del mundo del deporte español explicaba su actividad a los chicos, de manera sencilla y amistosa, y realizaba unos ejercicios de exhibición: Manuel Santana, en tenis; Emiliano, en baloncesto; Pirri, en fútbol; Miguel Torres, en natación; Mariano Díaz, como ciclista; Francisco Goyoaga, en hípica, entre otros muchos, participaron en este programa dirigido por José Castanyer. 
Adelante el inventor, presentado por Enrique Rubio, era un programa que aireaba el ingenio inventivo español: el inventor del enchufe eléctrico, el de una ventana especial, de un nuevo sistema musical, un sistema de seguridad que podía evitar caídas por el hueco del ascensor... en fin, artilugios de lo más variopinto. Un jurado exponía los inconvenientes o ventajas de la propuesta. Se trataba de un espacio para toda la familia, en horario infantil, que mostraba cómo algunos hombres trabajaban para resolver los problemas de los demás y sobre todo cómo el ingenio español (Blasco de Garay, Isaac Peral, Jaime Ferrán, Juan de Ochoa, etc.) había creado importantes inventos.

Para orientar profesionalmente a estudiantes se creó Protagonista el hombre, presentado por Federico Gallo y Clara Isabel Francia. El programa duraba 50 minutos y explicaba cómo llegar a ser aviador militar, ayudante técnico sanitario, sacerdote, camarero, bordadora, auxiliar de vuelo, técnico de turismo, etc. (AGA, Caja 35692). La mayoría de las propuestas se orientaron hacia la formación profesional que el franquismo se empeñó en potenciar y se dirigían a los chicos, porque todavía se pensaba que el destino de la mujer era el hogar.

Finalmente algunos programas infantiles de televisión asumieron el papel de orientadores del tiempo libre de los más jóvenes, como Lección de ocio que proponía actividades de todo tipo: teatro, encuadernación, la novela policiaca, cine amateur o libros de cocina.

Nuestro amigo el libro y Teatro de la juventud promovían la afición al teatro entre los jóvenes. En el primero, se representaba una versión de una obra clásica, realizada por Eloy G. De la Iglesia: Christian Andersen, Gustavo Adolfo Bécquer, Calderón de la Barca, Lope de Vega, Cervantes, Lafontaine, entre otros. Imagen club se encargó de lo mismo, pero con el cine, radio y televisión. No faltaron espacios dedicados a la música.

Muchos de estos programas tenían un elevado componente cultural y científico, como Amigos del espacio, quincenal, de treinta minutos de duración que pretendía canalizar la afición a las técnicas aeroespaciales de los muchachos, bajo la dirección y la realización de José Castanyer. En la primera parte, la familia Tungsteno, formada por el padre y tres hijos: Reinaldo, Taina y Leif visitaban una estación de radio enlace, el observatorio astronómico nacional, el Instituto Nacional de Meteorología, Telefónica, Iberia, la Junta de Energía Nuclear o la Escuela de Ingenieros Aeronáuticos. Allí su padre (un actor) o profesionales del lugar les explican el funcionamiento. Ni resultaba ameno, ni las explicaciones fáciles de entender. En la segunda parte, los presentadores en el estudio -Ana María Paso y José Antonio Rosa- leían la correspondencia enviada por los socios del Club infantil y juvenil de TVE.

Aventura, presentado por Luis Pruneda, se dedicó sin embargo, a profundizar en la espeleología o el alpinismo; y en Aventura juvenil una pandilla de chicos y chicas realizaban diversas actividades, mientras una voz en off las explicaba: excursión por el campo, pesca, baño en un río, montar en bici, etc.

Cabe también destacar en estos años la presencia del formato de contenedor: Antena infantil, El rincón de los chicos, Ven a jugar con nosotros o Formato 13 por 18. Ofrecían noticias, cine, música, canciones, magia, cuentos, dibujos animados, concursos, aventuras y estaban dirigidos para chicos y chicas entre 7 y 11 años, excepto 
Formato 13 por 18 que pretendía llegar a un público más mayor. Las variedades casi desaparecieron de la programación. Sólo se mantuvo Día de fiesta (1965-1969), también con las marionetas de Herta Frankel, las canciones de Gustavo Re y concursos con Juan Viñas.

\section{Conclusiones}

En TVE, durante el franquismo, hubo programas infantiles, pero no una programación infantil. Con la creación de la separata Vamos a la cama, se estableció el límite de la presencia de los niños como espectadores, pero nada más: siguieron existiendo programas para adultos en el horario de tarde. Esto da idea de que los programadores pensaban más en una audiencia familiar que infantil y juvenil: en ofrecer ternura, sonrisa y amabilidad para todos. La razón es la existencia de una única cadena de televisión que debía servir a todos los españoles y cuyos contenidos debían ser generalistas. Es cierto que en 1966 comenzó oficialmente la segunda cadena (UHF), pero tardó en extenderse y siempre tuvo un carácter minoritario (Palacio, 2005: 123-142).

No quiere decirse que no hubo preocupación por la audiencia infantil. Desde las primeras emisiones se incluyeron espacios destinados a este público. Los días y las horas de emisión estuvieron en función de la actividad escolar, después de las retransmisiones deportivas para los adultos. Ahora bien, a pesar de los esfuerzos de TVE por crear programas para niños y jóvenes, éstos preferían las series del oeste norteamericanas (Revista Española de la Opinión Pública, nº 2, 1965:214).

Al principio la programación radiofónica sirvió de inspiración: variedades, narraciones de cuentos y concursos. Luego llegaron los formatos de otros países (Italia y Estados Unidos). No faltaron tampoco los guiones originales, pero muchos copiaron los programas para adultos que, convenientemente adaptados, se etiquetaban para los pequeños. Los niños eran considerados gente menuda, adultos pequeños, y se les atribuían problemas -pequeños- y preocupaciones -también pequeñas- como tenían los mayores.

Primero hubo distinción por género, sobre todo en las aventuras seriadas, a partir de 1965, después por grupos de edad: para los más pequeños (3-6 años) se produjeron programas educativos (Jardilín) con el fin de enseñarles lo que estaba bien y lo que estaba mal; y para los más mayores (15-18 años) espacios para debatir sus problemas, donde un adulto moderaba y reconducía los temas según consideraba conveniente. Se hizo un acercamiento a lo que se creía que era su realidad de manera tan seria y dirigista que fracasó.

La participación de los niños en los programas fue evolucionando. Al principio, era muy limitada: se mantenían inmóviles dentro (en el estudio como espectadores) y fuera (en sus casas). Pero enseguida comenzó su intervención, primero respondiendo peguntas en concursos, luego de forma más activa colaborando en diálogos y debates (La vida y el juego, 1963). Fue muy relevante la creación del Club infantil de TVE no sólo porque muestra que la televisión fue consciente, en un determinado momento, de que el público infantil estaba ahí, sino también porque, a través del mismo, se canalizó la participación de los niños y de los jóvenes desde sus casas: mandaban dibujos, votaban, solicitaban canciones, etc. Y lo más importante, todas estas acciones tenían presencia en la pequeña pantalla, es decir, representaban actos públicos. 
Los diferentes tipos de producciones coincidieron en el intento de adoctrinar a los niños como buenos ciudadanos con sentimientos patrióticos y religiosos. El catolicismo estaba presente de forma directa en programas diseñados para su enseñanza o indirecta en todos y cada uno de los formatos, a través de parábolas, enseñanzas, recomendaciones, celebraciones, preguntas en concursos, etc. El patriotismo por su parte se divulgó sobre todo a través de vidas de españoles, del presente o del pasado, cuya trayectoria profesional o humana debía constituir un referente. No faltaron referencias que mostraban que lo español -inventos, deporte, música- triunfaba por todo el mundo.

Estos espacios realizaron una labor importante en la creación de aficiones y hobbies, destacando la lectura y la difusión de autores clásicos, no sólo españoles, sino también extranjeros. Es cierto que muchas de las obras fueron adaptaciones, pero los guiones respetaron en esencia el texto original.

La divulgación cultural, o al menos el repaso del bachillerato, fue otra de las constantes en algunos de los programas infantiles, aunque predominara, como se ha visto, la idea de entretener. Esta labor se realizó sobre todo a través de los concursos. Es importante señalar que no competían de forma individual, sino adscritos a colegios. Se fomentaba así la cooperación entre los miembros de cada equipo, perteneciente a un grupo escolar, y la rivalidad con el contrincante.

TVE difundió sobre todo elementos de la cultura popular en cuentos, juegos y música especialmente. Las historias ambientadas en épocas lejanas predominaron sobre las que mostraban el entorno próximo de los niños. Se mantuvo por lo tanto, también en los programas infantiles y juveniles, la táctica del franquismo de evadir, no de movilizar.

\section{Referencias}

BAGET, Josep María (1993): Historia de la Televisión en España (1956-1975). Barcelona, Feed-BacK.

BETHENCOURT, Tomás (1996): "Del directo al color. Una visión tecnológica de la televisión en España". Archivos de la Filmoteca, n 23-24, junio-octubre de 1996, pp.55-69.

BONAUD, Joseba (2008): "La influencia de la programación deportiva en el desarrollo histórico de TVE durante el monopolio de la televisión pública" (19561988), en Comunicación y Sociedad, vol. XXI, nº 1, pp. 103-136.

CARRERAS LARIO, Natividad (2011): "Los primeros programas de variedades de TVE: de La Hora Philips a Escala en Hi-Fi”. Revista Comunicación, $n^{\circ}$ 9, vol. 1, pp. 19-33.

DÍAZ, Lorenzo (1994): Historia de la televisión en España 1954-1995. Madrid, Alianza.

FERNÁNDEZ, Lluís (2007): El anuncio de la Modernidad. Estudios Moro 19551979. Valencia, Diputación de Valencia.

GARCÍA JIMÉNEZ, Jesús (1980): Radiotelevisión y política cultural en el franquismo. Madrid, CSIC. 
GONZÁLEZ, Manuel María (2011): Programas infantiles y juveniles de Televisión española (1956-1986): un catálogo para la investigación. Madrid, UCM, inédito.

GRANADOS GARÍN, Carlos (1966): "El teatro infantil y juvenil, en relación con los instrumentos de comunicación social". Ponencia presentada en el I Congreso de Teatro para la Infancia y la Juventud. Diciembre de 1966. Consultado en 22 de diciembre de 2012 en: http:/www.cervantesvirtual.com/controladores/ busqueda_facet.php? $\mathrm{q}=\mathrm{Granados}+\mathrm{Gar} \% \mathrm{C} 3 \% \mathrm{ADn}$

HERNÁNDEZ, Sira (2008): La historia contada en televisión. El documental televisivo de divulgación histórica en España. Barcelona, Gedisa.

ORTEGA, María Luisa y ALBERTOS, Ana (1998): “La ciencia en televisión española: primeros acercamientos a la divulgación”, en Secuencias, no 8, pp. 61-74.

PALACIO, Manuel (2005): Historia de la televisión en España. Barcelona, Gedisa.

Revista española de la Opinión Pública, $\mathrm{n}^{\circ}$ 1, mayo-agosto, 1965; nº 2, septiembrediciembre, 1965; $\mathrm{n}^{\circ} 3$, enero-marzo, 1966.

\section{Archivos}

Archivo General de la Administración (AGA)

\section{Anexo: Programas infantiles y juveniles de TVE (1958-1968)}

\begin{tabular}{|l|c|}
\hline $\begin{array}{l}\text { Programas infantiles y juveniles de TVE (1958-1968) } \\
\text { por orden cronológico de aparición }\end{array}$ & Periodo de inicio y fin \\
\hline 1. Primer aplauso & $01 / 01 / 58-09 / 01 / 66$ \\
\hline 2. Hazañas de Marianín y Teresa & $09 / 01 / 58-24 / 04 / 58$ \\
\hline 3. Premio al más listo, concurso para la gente menuda & $09 / 01 / 58-23 / 04 / 59$ \\
\hline 4. El circo & $19 / 01 / 58-29 / 10 / 60$ \\
\hline 5. Lo que cuenta la tía Cristina & $12 / 10 / 58-30 / 11 / 58$ \\
\hline 6. Lecciones de catecismo & ¿? $-08 / 01 / 59$ \\
\hline 7. No apto para mayores & $03 / 05 / 59-19 / 09 / 59$ \\
\hline 8. Teatro infantil & $10 / 05 / 59-25 / 12 / 74$ \\
\hline 9. Lecciones de cosas & $01 / 08 / 59-19 / 09 / 59$ \\
\hline 10. A la rueda, rueda & $29 / 10 / 59-28 / 05 / 60$ \\
\hline 11. Gente menuda & $22 / 11 / 59-27 / 12 / 59$ \\
\hline 12. La aventura de la música & $29 / 12 / 59-25 / 09 / 65$ \\
\hline 13. Tiovivo & $03 / 01 / 60-22 / 10 / 60$ \\
\hline 14. Herta Frankel y sus marionetas & $21 / 02 / 60-18 / 09 / 60$ \\
\hline 15. Walter y la familia corchea & $04 / 08 / 60-15 / 09 / 60$ \\
\hline 16. Rumbo sur & $30 / 09 / 63-25 / 05 / 64$ \\
\hline 17. Recreo & $08 / 10 / 60-24 / 09 / 64$ \\
\hline 18. Sobresaliente & $16 / 10 / 60-28 / 05 / 61$ \\
\hline 19. Cuento infantil & $22 / 12 / 60-29 / 01 / 61$ y de \\
\hline 20. Las marionetas Herta Frankel & $01 / 10 / 65-11 / 02 / 66$ \\
\hline
\end{tabular}




\begin{tabular}{|c|c|}
\hline $\begin{array}{l}\text { Programas infantiles y juveniles de TVE (1958-1968) } \\
\text { por orden cronológico de aparición }\end{array}$ & Periodo de inicio y fin \\
\hline 21. Chavales & $08 / 06 / 61-30 / 09 / 65$ \\
\hline 22. Muchachos al aire libre & $06 / 08 / 61-24 / 09 / 61$ \\
\hline 23. Torneo & $25 / 06 / 61-24 / 09 / 61$ \\
\hline 24. Sólo para menores & $06 / 01 / 62-24 / 09 / 66$ \\
\hline 25. Silla de pista & $03 / 06 / 62-26 / 09 / 63$ \\
\hline 26. Telepequediario & $06 / 10 / 62-28 / 09 / 63$ \\
\hline 27. Fiesta con nosotros & $28 / 10 / 62-26 / 09 / 65$ \\
\hline 28. Concertino & $03 / 07 / 63-13 / 02 / 64$ \\
\hline 29. Un mundo para los niños & $27 / 07 / 63-28 / 09 / 63$ \\
\hline 30. El barquito de papel & $04 / 10 / 63-19 / 09 / 64$ \\
\hline 31. Mundo ligero ( $\sin$ burbujas) & $04 / 10 / 63-24 / 09 / 64$ \\
\hline 32. La vida y el juego & $05 / 10 / 63-27 / 09 / 64$ \\
\hline 33. Visado para el futuro & $05 / 10 / 63-27 / 09 / 65$ \\
\hline 34. Lección de ocio & $01 / 10 / 64-25 / 02 / 65$ \\
\hline 35. A media voz & $10 / 03 / 64-05 / 07 / 65$ \\
\hline 36. Nuestro amigo el libro & $01 / 05 / 64-08 / 01 / 65$ \\
\hline 37. El club de los inventores & $01 / 10 / 64-08 / 10 / 64$ \\
\hline 38. Campeones & $03 / 10 / 64-01 / 06 / 65$ \\
\hline 39. Teatro de la juventud & $03 / 10 / 64-18 / 09 / 65$ \\
\hline 40. Aventura & $06 / 10 / 64-28 / 09 / 65$ \\
\hline 41. Adelante el inventor & $15 / 10 / 64-30 / 09 / 65$ \\
\hline 42. Santi, botones de hotel / Santi en el hotel & $06 / 03 / 65-01 / 05 / 65$ \\
\hline 43. Patio de corredor & $12 / 06 / 65-31 / 07 / 65$ \\
\hline 44. Cesta y Puntos & $01 / 10 / 65-29 / 05 / 71$ \\
\hline 45. Día de Fiesta & $03 / 10 / 65-20 / 04 / 69$ \\
\hline 46. Antena infantil & $07 / 10 / 65-24 / 04 / 71$ \\
\hline 47. El rincón de los chicos & $09 / 10 / 65-02 / 09 / 67$ \\
\hline 48. Escuela de campeones & $06 / 11 / 65-10 / 09 / 66$ \\
\hline 49. Las fronteras de la ciencia & $24 / 02 / 66-04 / 08 / 66$ \\
\hline 50. Juego y juguetes & $21 / 04 / 66-05 / 08 / 66$ \\
\hline 51. Ven a jugar con nosotros & $18 / 05 / 66-25 / 10 / 67$ \\
\hline 52. El cuarto de los niños & $27 / 09 / 66-04 / 10 / 66$ \\
\hline 53. Jardilín & $05 / 10 / 66-01 / 07 / 68$ \\
\hline 54. Protagonista, el hombre & $06 / 10 / 66-24 / 09 / 68$ \\
\hline 55. Cuál es tu final? & $08 / 10 / 66-03 / 06 / 67$ \\
\hline 56. Formato 13 por 18 , Formato 13 por 16 , Formato 13 por 13 & $08 / 10 / 66-13 / 10 / 67$ \\
\hline 57. Amigos del espacio & $14 / 02 / 67-27 / 12 / 68$ \\
\hline 58. El mar, ese mundo maravilloso & $18 / 05 / 67-20 / 09 / 68$ \\
\hline 59. Biblioteca juvenil & $24 / 06 / 67-16 / 06 / 69$ \\
\hline $\begin{array}{l}\text { 60. Habla contigo/Habla contigo Jesús Urteaga } \\
\text { / Habla el Padre Urteaga }\end{array}$ & $14 / 10 / 67-10 / 06 / 70$ \\
\hline
\end{tabular}




\begin{tabular}{|l|c|}
\hline $\begin{array}{l}\text { Programas infantiles y juveniles de TVE (1958-1968) } \\
\text { por orden cronológico de aparición }\end{array}$ & Periodo de inicio y fin \\
\hline 61. Misión rescate & $19 / 10 / 67-11 / 11 / 78$ \\
\hline 62. Objetivo una inquietud & $20 / 10 / 67-10 / 11 / 67$ \\
\hline 63. Antoñita la fantástica & $21 / 10 / 67-24 / 12 / 67$ \\
\hline 64. Todos somos jóvenes & $21 / 10 / 67-21 / 09 / 68$ \\
\hline 65. Aventuras de Mumú & $11 / 11 / 67-04 / 05 / 68$ \\
\hline 66. Tenemos la palabra & $02 / 12 / 67-07 / 12 / 68$ \\
\hline 67. Imagen club & $30 / 12 / 67-06 / 04 / 69$ \\
\hline 68. La tortuga presurosa & $09 / 02 / 68-25 / 09 / 68$ \\
\hline 69. Concurso juvenil de magia & $08 / 06 / 68-27 / 09 / 68$ \\
\hline 70. Nuestra guitarra & $12 / 06 / 68-19 / 09 / 69$ \\
\hline 71. Aventura juvenil & $02 / 07 / 68-24 / 09 / 68$ \\
\hline 72. Clarín & $08 / 07 / 68-30 / 09 / 68$ \\
\hline 73. Clan familiar & $13 / 07 / 68-18 / 10 / 69$ \\
\hline 74. Festival de canciones infantiles & $03 / 10 / 68\left(2^{\mathrm{a}}\right.$ edición) - 21/11/68 \\
\hline 75. Telecomedia infantil y juvenil & $08 / 10 / 68-05 / 11 / 68$ \\
\hline 76. La solución... mañana & $24 / 10 / 69-18 / 04 / 70$ \\
\hline 77. Vuestro amigo Quique & $24 / 06 / 72-23 / 02 / 74$ \\
\hline
\end{tabular}

